\title{
Bilateral Bispectral index (BIS) monitoring on epileptiform activity detection: an anti-NMDA encephalitis case
}

Marta Carvalho1; Patrícia Ramos ${ }^{1}$; Raquel Monte

1- Centro Hospitalar do Porto

\section{INTRODUCTION}

- Anti-N-methyl-D-aspartate receptor (NMDAR) encephalitis is a disorder characterized by acute behaviour and psychiatric changes, seizures, speech disorders and disautonomia.

- Despite the severity of the disorder, the prognosis is usually good.

- Bilateral Bispectral Index (BIS) monitoring displays four channels of electroencephalogram (EEG) and also allow monitoring density spectral array (DSA).

\section{CASE REPORT}

18 year-old male;

$\square$ No relevant medical history.

\section{1 month}

Neuropsychiatric changes and speech disorder;

Autonomic dysfunction;

$\checkmark$ Hypoventilation periods;

$\checkmark$ Refractory seizures.

Anti-NMDA antibodies in the blood and cerebrospinal fluid (CSF) positive<smiles>C1=CC=C1</smiles>

Autoimmune anti-NMDA encephalitis

Depression of level of consciousness and severe hypoventilation

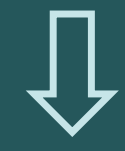

ICU

Standard monitoring of ICU;

Bilateral bispectral index (BIS) monitor;

Plasmaferesis.

No recurrence of epileptiform discharges or a predominant high frequency DSA pattern;

Clinical evolution during hospitalization was favourable with totally recovery on follow-up.

\section{Day 2 after admission}

Episodes of hyperthermia, with no identifiable infectious source

DSA pattern showed an abrupt change with increase density in high frequencies domain;

BIS monitor also revealed epileptiform discharges, similar to the ones on conventional EEG

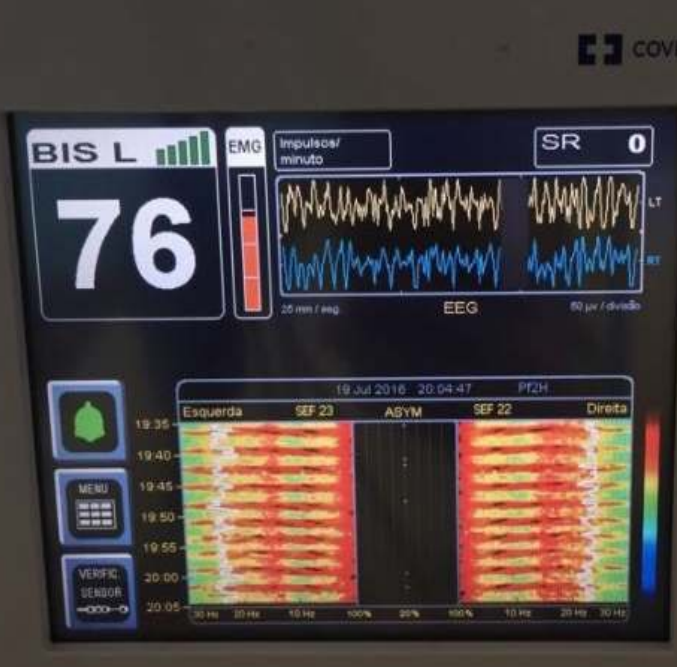

Tiopental bolus was administered

Conventional EEG performed afterwards was compatible with electrical status epilepitucus;

Sedative and anti-epileptic medications were adjusted;

Succeeding reductions in sedative an analgesic medications was perform according to DSA monitoring and conventional EEG results.

\section{DISCUSSION}

- Continuous EEG monitoring of this patients is highly desirable, however resource unavailability limits its use;

- On the other side, EEG interpretation is complex, which lead to development of quantitative analysis based algorithms, such as processed EEG and density spectral array;

- In this case, identification of a DSA pattern compatible with epileptiform activity was decisive on patient's treatment and prognosis.

\section{LEARNING POINTS}

Bilateral BIS monitoring can be an adjuvant tool on epileptiform activity diagnosis, especially when continuous EEG is not available. 